\title{
Asociacionismo vecinal en las ciudades medias andaluzas
}

Javier ESCALERA REYES*

Esteban RUIZ BALLESTEROS*

\section{RESUMEN}

Este artículo caracteriza el asociacionismo vecinal en las ciudades medias andaluzas (entre 20 mil y 50 mil habitantes) haciendo especial hincapié en los rasgos fundamentales de su funcionamiento interno, así como en las formas y el grado de su articulación externa. A continuación explora la relevancia de dos factores que entendíamos potencialmente significativos para comprender la diversidad interna en el asociacionismo vecinal: (1) las diferencias/similitudes socio-económico-culturales de las localidades estudiadas, y (2) las formas y signos del gobierno local en cada municipio. Con todo ello pretendemos una aproximación a las asociaciones de vecinos andaluzas y a los factores que nos ayuden a comprender su carácter y papel en la vida social, cuestiones potencialmente trasladables a otros contextos y asociacionismos.

\section{PALABRAS CLAVE}

Asociacionismo, asociacionismo vecinal, política local.

* GISAP 


\section{ABSTRACT}

In this paper we characterize the neighbourhood associations in the Andalusian medium size cities (between 20 thousand and 50 thousand inhabitants) doing special emphasis in the fundamental characteristics of its internal operation as well as in the forms and the degree of its external social articulation. Subsequently we explore the importance of two factors that we think potentially significant to understand the internal diversity in the Andalusian neighbourhood associations: (1) the socio-economic and cultural differences and similarities among the studied cities, and (2) the forms and signs of the local government in each municipality. With all this elements we intend an approximation to the Andalusian neighbourhood associations and to the factors that help us to understand their character and role in the social life, potentially transferable questions to other contexts and associationisms.

\section{KEYWORDS}

Associationism, neighbourhood associationism, local politics

\section{EL ASOCIACIONISMO VECINAL EN ANDALUCÍA Y SU CONSIDERACIÓN COMO OBJETO DE ESTUDIO}

El asociacionismo andaluz sigue adoleciendo de la falta de un conocimiento en profundidad que, más allá de los estereotipos, permita evaluar su significación social y sus potencialidades como elemento articulador y dinamizador de nuestra sociedad civil1. Pero si ello es cierto en general, particularmente escaso — salvo algún estudio de caso (Ruiz y Páez, 1997; Navarro, 1999; Del Río y Coca, 2003)— es el conocimiento que tenemos sobre el asociacionismo vecinal en sus diferentes facetas, campos, categorías y tipos. Este desconocimiento, paradójicamente, contrasta con el protagonismo que, al menos a nivel formal, detenta el asociacionismo vecinal en el ámbito de la política institucional, apareciendo junto a sindicatos y patronales como los agentes sociales oficiales propiciadores de legitimidad democrática.

${ }^{1}$ Para un estado de la cuestión sobre los estudios sobre asociacionismo en Andalucía ver: Escalera, 1988, 2001b y 2002. 
Transcurridos casi treinta años desde los momentos de regeneración del movimiento vecinal, y reproduciendo en términos generales lo que parece ser su situación generalizada en el conjunto del Estado español2 ${ }^{2}$ el asociacionismo vecinal andaluz actual presenta rasgos muy marcados que ya empezaban a apuntarse a principio de los años ochenta, una vez culminada la denominada transición, y que, en términos generales, podríamos definir como desarticulación y desactivación. Las asociaciones de vecinos (en adelante AA. VV.), que jugaron un papel fundamental en las movilizaciones socio-políticas que determinaron la recuperación de la Democracia, a nivel del conjunto del Estado, y la conquista de la autonomía plena, en el caso concreto de Andalucía, han perdido mucho de su protagonismo y del papel de motor de la participación ciudadana en la vida social y política de la mayor parte de nuestras ciudades y pueblos. Esta pérdida de significación real responde, en parte, a la existencia de otros canales y organizaciones que asumen las funciones que, en condiciones de falta de democracia o de debilidad de las instituciones y formaciones políticas democráticas, desempeñaron las AA. VV. Pero la explicación de la "normalización" del sistema político democrático no es suficiente para justificar la desaparición de asociaciones o la decadencia de muchas de ellas que han perdido arraigo y capacidad de movilización del vecindario. Tampoco se explica por la ausencia de los problemas y necesidades materiales, infraestructurales y de convivencia que en muchos casos motivaron su formación, problemas que no sólo no se han solucionado, sino que en algunos casos se han incrementado.

Las profundas transformaciones que ha experimentado la sociedad andaluza en estos últimos años constituyen una de las causas de la crisis del movimiento asociativo ciudadano. El crecimiento del individualismo influye poderosamente en la pérdida de la capacidad de convocatoria de estas asociaciones, salvo en situaciones muy puntuales de cara a problemas concretos que afectan directa y personalmente a la mayoría de los vecinos.

No obstante, uno de los factores que más han incidido en esta situación ha sido la actuación de las organizaciones políticas, de manera particular las autoidentificadas como de izquierda, que, una vez estabilizado el sistema democrático, acapararon para sí el papel de único canal y órgano de participación y acción política, actuando

\footnotetext{
${ }^{2}$ El monográfico de la Revista Documentación Social ( $n^{\circ}$ 94, 1994), dedicado al Mundo Asociativo, ofrece un panorama y balance que puede servir como referencia a pesar del tiempo transcurrido.
} 
abiertamente en la desactivación de las asociaciones en las que se apoyaron y de las que se sirvieron durante los tiempos en los que eran organizaciones ilegales o carecían de infraestructuras y militancia suficientes. Se produjo así una descapitalización de las AA. VV. a través de la captación de la mayoría de sus líderes y miembros más activos, para construir o enriquecer sus propias organizaciones y cuadros dirigentes.

Hoy son pocas las asociaciones vecinales que mantienen una vitalidad y participación relevantes. La mayoría de ellas son antiguas asociaciones que han conseguido reconvertirse $u$ otras nuevas que han surgido sobre bases y objetivos diferentes a los que primaban en la década de los 70 . Muchas han incorporado nuevos objetivos de carácter recreativo, cultural, deportivo, medioambiental, educativo, e incluso en bastantes ocasiones se han convertido en entidades dispensadoras de servicios no prestados por las administraciones públicas.

Desde esta perspectiva procesual y sociopolítica del asociacionismo vecinal, nuestra investigación ${ }^{3}$ se ha ocupado del asociacionismo vecinal en las 40 ciudades andaluzas de entre 20.000 y 50.000 habitantes, según el Padrón de 1998.

\begin{tabular}{|l|l|l|l|l|l|l|l|l|}
\hline A0 localidades andaluzas entre 20.000 & $\mathbf{5 0 . 0 0 0}$ habitantes & \\
\hline Adra & 25.016 & Alcalá la Real & 21.493 & Almuñécar & 20.997 & Andújar & 38.254 \\
\hline Antequera & 40.239 & Arcos de la Fra. & 27.897 & Baena & 20.057 & Barbate & 21.916 \\
\hline Baza & 20.113 & Benalmádena & 28.479 & Cabra & 20.819 & Camas & 25.499 \\
\hline Carmona & 25.326 & Coria del Río & 23.517 & Écija & 37.113 & Estepona & 39.178 \\
\hline Fuengirola & 44.924 & Guadix & 20.322 & La Rinconada & 26.942 & Lebrija & 23.924 \\
\hline Loja & 20.143 & Los Palacios & 32.114 & Lucena & 35.564 & Mairena del Aljarafe & 31.793 \\
\hline Martos & 22.391 & Mijas & 37.490 & Montilla & 22.792 & Morón de la Frontera & 28.232 \\
\hline Priego & 22.196 & Puente Genil & 27.918 & Puerto Real & 33.415 & Rincón de la Victoria & 20.629 \\
\hline Ronda & 33.806 & Roquetas de Mar & 40.582 & Rota & 24.704 & San Juan Aznalfarache & 20.563 \\
\hline San Roque & 22.322 & Torremolinos & 37.235 & Úbeda & 32.524 & Utrera & 45.947 \\
\hline
\end{tabular}

Fuente: INE. Revisión del Padrón Municipal de Habitantes 1998.

\footnotetext{
${ }^{3}$ La investigación ha sido posible gracias a una subvención de la Consejería de Relaciones Institucionales de la Junta de Andalucía y su trabajo de campo fue desarrollado durante el año 2000. El equipo de investigación estuvo compuesto por los profesores Agustín Coca Pérez y Macarena Hernández Ramírez de la Universidad Pablo de Olavide, Reyes García del Villar Balón de la Universidad de Córdoba y Ángel del Río Sánchez del GISAP. El diseño de la base de datos y la explotación de la misma estuvo a cargo de Pedro J. Pérez Moreno.
} 
Todas ellas suman una población de 1.144.385 habitantes, lo que supone el 15 '4\% de la población total andaluza. Este dato cuantitativo es ya suficientemente representativo, pero adquiere especial significación si consideramos la relevancia de este tipo de ciudades medias desde el punto de vista territorial, socioeconómico, cultural, identitario,... para el conjunto de Andalucía (Cano y García; 2000; Escalera y Martín, 1990, Feria, 1990 y 1992; López Chacón, 1990; Zoido, 1990). Si bien tradicionalmente la mayoría de estas ciudades respondían al modelo de agrociudad (López Casero, 1989), en la actualidad presentan nuevas configuraciones, como la de ciudades dormitorio de las áreas metropolitanas (San Juan de Aznalfarache, Mairena del Aljarafe...), centros turísticos (Torremolinos, Fuengirola, Estepona...), núcleos industriales y de servicios (Lucena, Puerto Real...), lo que ha introducido una notable diversificación interna que responde a la propia heterogeneidad del territorio y la sociedad andaluza.

Nuestro objetivo en este texto es caracterizar de manera general el asociacionismo vecinal en las ciudades medias andaluzas. A partir de aquí, analizaremos el peso de dos factores potencialmente relevantes para entender la diversidad del asociacionismo: (1) los condicionantes socio-económicos-culturales, y (2) las formas y signos del gobierno local en cada municipio. El primero de ellos tiene que ver con nuestra visión del asociacionismo en general, y el vecinal en particular, como un elemento profundamente imbricado con los factores sociales, económicos y culturales (Carmona y Collado, 1994; Escalera, 2002). El segundo, con nuestra consideración del asociacionismo como un elemento destacado en la política local (Ruiz, 2000; Ruiz y Páez, 1997).

Hemos desarrollado en cada localidad y en referencia a cada asociación vecinal diversas estrategias de investigación. En primer lugar, un estudio -basado en la información secundaria disponible- de los datos básicos que caracterizan, según diferentes magnitudes, a las distintas localidades. Posteriormente, un conjunto de entrevistas con informantes privilegiados que nos han proporcionado una primera visión del estado, evolución y dimensiones del fenómeno asociativo vecinal a nivel local. A partir de aquí hemos llevado a cabo un proceso de observación y seguimiento directo de cada una de las AA. VV., contrastando las informaciones recibidas, lo que nos ha permitido tanto la actualización de los datos disponibles, como la generación de información descriptiva de primera mano. El eje central del trabajo con las asociaciones lo han constituido las visitas a las sedes y las entrevistas a los directivos 
y líderes vecinales. Toda la información así recogida se ha traducido posteriormente a un guión homogéneo, sintético y codificado que ha permitido el tratamiento estadístico de los items considerados más relevante a tenor de nuestros intereses. De este modo, la ejecución completa del proyecto de investigación nos ha reportado una etnografía de los sistemas asociativos vecinales en cada localidad, así como una base estadística ${ }^{4}$ para realizar análisis a nivel del conjunto de las ciudades medias andaluzas. Como vemos, las perspectivas cuantitativa y cualitativa podrán articularse de forma recursiva.

Quizá el elemento distintivo más importante de nuestra investigación, y la base que valida tanto el origen de los datos que manejamos como su rigurosidad y el análisis que hacemos de los mismos, es el carácter del propio trabajo de campo. Nuestra recogida de datos no ha utilizado herramientas indirectas, como la encuesta telefónica o el cuestionario postal, ni siquiera se ha implementado una encuesta cerrada, sino que hemos utilizado la entrevista cara a cara — guión abierto- con los directivos y líderes de las AA. VV., así como la observación de la vida asociativa. Por tanto, hemos recogido los datos desde una perspectiva cualitativista y ha sido posteriormente el propio equipo el que ha traducido esa información a un formato susceptible de tratamiento cuantitativo. Con ello no obligamos a los informantes a traducir sus opiniones y visiones a nuestros códigos, sino que somos nosotros mismos los que debemos hacer esa traducción. Esta estrategia nos garantiza una mayor homogeneidad de la propia información codificada, pero no está exenta de problemas. En primer lugar sólo es factible si el equipo cuenta con investigadores experimentados (tanto en el trabajo de campo como en las fases de análisis), y por otro lado supone una extrapolación de técnicas factibles en universo de estudio reducido (estudios de comunidad) a ámbitos de investigación mucho más amplios como es el caso de éste. En ambos casos nos parece que hemos superado relativamente las adversidades —sobre todo en el primero—, aunque habrá que perfilar mucho más este tipo de estrategias.

A continuación presentamos los resultados que nos permiten caracterizar el asociacionismo vecinal en las ciudades medias andaluzas en sus aspectos más

\footnotetext{
${ }^{4}$ El tratamiento estadístico comportó un análisis descriptivo (descripción de los items para el total de la muestra y descripción de items por niveles de dos variables), análisis comparativo (comparaciones, paramétricas o no paramétricas, de diversas variables) y análisis multivariado (análisis de correspondencias múltiples, tipologías o clases latentes, con diversos cortes).
} 
sobresalientes. Posteriormente establecemos una comparación entre estos resultados generales y los que obtenemos si segmentamos las asociaciones estudiadas con relación a su ubicación territorial (condicionante socio-económico-cultural) o por el carácter del gobierno local de la ciudad donde operan. Todo esto nos permite concluir sobre la relevancia o no de estos dos factores tan dispares para comprender la situación del asociacionismo vecinal en las ciudades medias andaluzas.

\section{CARACTERIZACIÓN GENERAL}

Uno de los elementos que más han marcado tradicionalmente nuestro conocimiento sobre el asociacionismo ciudadano en general, y el vecinal en particular, ha sido precisamente la dificultad para determinar su propia existencia: número real de asociaciones, ubicación... Bien es verdad que el notable dinamismo que caracteriza a estas formas de expresión formalizada de la sociabilidad cívica —con frecuentes apariciones y desapariciones de asociaciones-y su proliferación, dificulta notablemente la tarea de disponer de registros que se adecuen a la realidad del propio fenómeno y que nos puedan permitir en todo momento tener una información apropiada y rigurosa sobre su dimensión y localización. Por eso consideramos que uno de los resultados más relevantes de nuestro estudio es la corroboración efectiva de la muy deficiente información de la que las instituciones públicas y los investigadores disponemos a la hora de referirnos a un fenómeno tan importante para la articulación y cohesión social de nuestras ciudades como es el asociacionismo vecinal. Esta investigación ha supuesto una actualización crítica de la información disponible sobre las AA. VV. de los municipios medios andaluces. Merece la pena que nos detengamos someramente en esta circunstancia.

Nuestro punto de partida fue el Registro Oficial de Asociaciones del que dispone la Consejería de Gobernación de la Junta de Andalucía. Dicho registro ha sido rigurosamente contrastado con los correspondientes Registros Municipales, en el caso de que éstos existieran. Y finalmente, la información facilitada por ambos registros ha sido minuciosa y exhaustivamente comprobada mediante un trabajo de campo para la localización efectiva de esas asociaciones en sus respectivos ámbitos de influencia. Sólo este último tipo de rastreo puede acercarnos eficazmente a un dimensionamiento real del asociacionismo vecinal. De las $682 \mathrm{AA}$. VV. registradas municipal o autonómicamente, tan sólo un $56,1 \%$ existen como tales, lo cual evidencia que las instituciones públicas manejan unos datos claramente superiores a los que 
la práctica social reconoce. No hace falta abundar en las consecuencias que esta deficiencia informativa tiene para establecer valoraciones de cualquier tipo sobre el tejido asociativo vecinal en la Comunidad Autónoma andaluza. Los registros locales son, lógicamente, los que se adecuan más a la realidad que hemos contrastado, pero aun así hay que destacar que mediante nuestro trabajo de campo hemos encontrado asociaciones activas que no se hallaban recogidas en ningún registro oficial (un $11 \%$ del total). Sin duda, todo ello supone una notable matización a la dimensión del asociacionismo vecinal en Andalucía, y más concretamente en las localidades que hemos estudiado.

Después de todas estas puntualizaciones podemos ofrecer un primer dato ilustrativo del fenómeno asociativo vecinal en los municipios medios andaluces: 2.988 habitantes por asociación de vecinos. Este índice no es una mera operación matemática a partir de información secundaria disponible, sino que se deriva de una rigurosa constatación empírica del asociacionismo realmente existente.

Nuestra caracterización general del movimiento asociativo vecinal tiene en cuenta dos factores principales: la ubicación del ámbito de acción de las asociaciones y el momento de su constitución. Con respecto al primero hay que apuntar que el 65,9\% de las AAVV responde a contextos vecinales enmarcados en los núcleos principales de población de los municipios estudiados, y el 34\% restante corresponde a AA. VV. que se ubican fuera de las cabeceras de los municipios (un 11,5\% son asociaciones de entidades locales menores, el 15,2\% responde a barriadas rurales y el 7,3\% a urbanizaciones alejadas de los respectivos núcleos principales). En este punto es necesario señalar la confusión creciente —en la que cae la propia administración a tenor del contenido de sus propios registros - entre AA. VV. y comunidades de propietarios. Esta circunstancia no es baladí, ya que es muestra indirecta del sesgo que el propio asociacionismo vecinal y su consideración administrativa están sufriendo, basculando, en algunos casos, casi de manera exclusiva hacia los intereses de propietarios preocupados por cuestiones urbanísticas y de servicios que afectan a sus viviendas. Este fenómeno ha sido detectado principalmente en las urbanizaciones que proliferan en la periferia de las poblaciones 0 al hilo de vías de comunicación importantes. Estas comunidades de propietarios no son realmente AA. VV., y creemos que ni a nivel analítico, ni a nivel administrativo debieran confundirse uno y otro tipo de asociacionismo, por muy próximos que puedan parecer. El caso es que, tanto la administración autonómica como la local —aunque en menor medida-, incluyen en sus registros (y a veces también en sus políticas) a unas y otras de manera indistinta, 
desvirtuando un modelo de asociacionismo ciudadano clásico con otro que responde a una concepción del ciudadano como cliente consumidor de servicios públicos sobre la base de supuestos derechos de propiedad.

En cuanto al momento de aparición, podríamos dividir a las asociaciones estudiadas en tres grupos. En el primero estarían las asociaciones surgidas con anterioridad a 1981, que representan el $11,1 \%$ del total. En el segundo recogemos a las AA. VV. aparecidas entre 1981 y 1990, que suponen un 30,4\% de las existentes. Y por último, las asociaciones fundadas después de 1990 que conforman un total del $57,8 \%$. Como vemos, más de la mitad de las actuales AA. VV. tiene menos de diez años, y las que superan los veinte años de existencia apenas pasan del $10 \%$. Poco parece quedar de aquel movimiento vecinal histórico al que nos referíamos en nuestra introducción. Por su cronología, el asociacionismo vecinal de las ciudades medias andaluzas es básicamente un fenómeno nuevo, que surge con la consolidación del estado de derecho: es consecuencia del mismo antes que su causa.

Íntimamente unido a este último aspecto de la constitución de las AA. VV. está el motivo aducido para su creación. En este caso los factores mayoritarios son las reivindicaciones sobre temas relacionados con urbanismo y vivienda $(40,1 \%)$, cuestión ésta que ha sido considerada tradicionalmente uno de los campos prioritarios de los movimientos vecinales, y en menor medida $(24,1 \%)$ la formalización asociativa planteada como medio para dotar de servicios al barrio. Es curioso cómo determinadas preocupaciones y actividades que en principio no tuvieron un papel preponderante entre los motivos de aparición, posteriormente han alcanzado una gran importancia en el funcionamiento de estas AA. VV., como veremos más adelante.

\begin{tabular}{|l|c|}
\hline \multicolumn{2}{|c|}{ Motivos de aparición } \\
\hline Servicios al barrio & $24,1 \%$ \\
\hline Urbanismo/vivienda & $40,1 \%$ \\
\hline Verbenas/fiestas & $8,3 \%$ \\
\hline Seguridad & $4,3 \%$ \\
\hline Coordinación institucional & $12,3 \%$ \\
\hline Segregación/política & $3,2 \%$ \\
\hline Otros & $7,8 \%$ \\
\hline
\end{tabular}


En principio barajamos como hipótesis exploratoria el hecho de que existiese algún tipo de vinculación entre el momento de aparición de las AA. VV. y los motivos que indujeron a su creación. Una vez aplicada la correspondiente herramienta estadística, comprobamos que no existía vinculación significativa entre período de fundación/motivo de aparición, con lo cual no es posible caracterizar al asociacionismo vecinal en este sentido: los diferentes motivos de aparición se reparten a lo largo de los más de treinta años en cuyo lapso se sitúa cronológicamente el origen de las AA. VV. estudiadas.

De una manera casi unánime $(94,9 \%)$ las AA. VV. manifiestan que su creación fue iniciativa de un grupo de vecinos del barrio. En principio estos resultados llevan cuando menos a la reflexión en un campo asociativo muy joven. Aunque parece evidente la relación de ese boom asociativo de la última década con la puesta en marcha de políticas de fomento del asociacionismo en los diferentes ayuntamientos estudiados, ello no queda, sin embargo, reflejado en las manifestaciones de los propios vecinos y sus dirigentes, que vinculan el nacimiento de la práctica totalidad de éstas a una iniciativa vecinal independiente. Esta circunstancia, de nuevo, propicia una cierta paradoja cuando comprobamos el grado de dependencia que las AA. VV. tienen con respecto a las administraciones públicas, y más concretamente a la administración local, como se verá más adelante.

En este intento de caracterización general nos quedaría reflejar la actividad principal con la que las distintas asociaciones estudiadas se sienten comprometidas, y establecer una comparación entre esas actividades centrales que en la actualidad marcan la vida de las mismas y esas otras que en un pasado más o menos lejano motivaron la razón de su aparición. De esta manera podemos aventurar una suerte de evolución funcional del asociacionismo vecinal en los municipios medios de Andalucía.

\begin{tabular}{|l|c|}
\hline \multicolumn{2}{|c|}{ Actividad principal } \\
\hline Fiestas y celebraciones & $59,0 \%$ \\
\hline Servicios comunitarios & $41,8 \%$ \\
\hline información & $17,0 \%$ \\
\hline Acciones reivindicativas & $36,8 \%$ \\
\hline
\end{tabular}


No obstante, de nuevo, los datos estadísticos no corroboran con claridad ninguna vinculación que nos permita establecer líneas evolutivas claras en el movimiento vecinal. Existe una obvia derivación general hacia la organización de fiestas y celebraciones por parte del conjunto de AA. VV., pero esta es prácticamente una tendencia repartida proporcionalmente entre los diferentes motivos de aparición. Si acaso, tan sólo es destacable un mayor número de asociaciones que, habiendo sido constituidas con el objeto principal de prestar servicios al barrio, se han convertido con el paso de los años en AA. VV. que principalmente organizan fiestas y celebraciones. Lo que sí resulta evidente es la tendencia general hacia la organización de fiestas y celebraciones que presenta el conjunto de asociaciones existentes.

En nuestra investigación hemos querido también hacer siquiera una somera aproximación al posicionamiento de las AA. VV. en torno a Andalucía como entidad política y cultural, y a la conciencia de ello por parte de sus integrantes. Para eso nos fijamos de forma muy específica en el grado de implicación de las AA. VV. en las celebraciones referidas a Andalucía ( 28 de febrero - día oficial de la comunidad autónoma- y 4 de diciembre — conmemoración de carácter nacionalista-). Resulta sorprendente el escaso nivel de celebración andalucista del movimiento vecinal en las ciudades medias, sobre todo si tenemos en cuenta que las celebraciones y fiestas son la actividad principal declarada de muchas de las AA. V. Tan sólo el $18,8 \%$ de las AA. WV. celebra de forma autónoma algún tipo de acto con motivo del 28 de febrero. En cuanto a la celebración del 4 de diciembre, su significación es prácticamente nula: sólo el $0,5 \%$ de las AA. VV. realiza algún acto conmemorativo. Siendo las fiestas y celebraciones una de las actividades prioritarias de las AA. VV. estudiadas, resulta muy elocuente la priorización que se hace con los potenciales referentes festivos: las fiestas de las AA. VV. tomarán como objeto al propio barrio, obviarán al ámbito local como tal —hasta aquí coherente-, pero también se obvia al referente andaluz. Estas circunstancias simbólico-festivas están indicando con claridad un modelo de articulación política más amplio, del que las AA. VV. son parte integrante.

Una vez que hemos prestado atención a los elementos más generales que conformarían una caracterización global de las AA. VV., vamos a centrarnos en torno a dos ámbitos que nos ayudarán a entender su funcionamiento y su articulación externa. 


\subsection{Sobre el funcionamiento asociativo}

El desarrollo tanto de actividades como de una vida asociativa regular está notablemente determinado por la disponibilidad de locales e instalaciones que permitan reunirse a los socios y directivas, así como organizar en ellos (si reúnen las condiciones, tamaño e infraestructura adecuadas) actividades de muy diversa índole, dirigidas al conjunto del vecindario. Por tanto, la disponibilidad de local es un elemento estratégico para aproximarnos al funcionamiento asociativo. Si bien su existencia no garantiza una vida asociativa dinámica, con actividades variadas y oferta múltiple, su inexistencia a buen seguro dificulta incluso el propio funcionamiento ordinario, al no poderse garantizar siquiera una mínima infraestructura, como un archivo estable, por ejemplo.

Tan sólo el 48,6\% de las asociaciones disponen de local, lo cual nos deja a más de la mitad sin instalaciones de las que disponer y por tanto con una importante merma potencial en sus actividades. De esas asociaciones que sí disponen de local, sólo el $17,8 \%$ lo disfruta en propiedad, ya que el $13 \%$ lo tiene alquilado y el $69,2 \%$ lo disfruta en forma de cesión, normalmente por parte de los ayuntamientos. No podemos obviar este punto de partida a la hora de reflexionar sobre el funcionamiento asociativo, ya que los datos nos indican a las claras dos circunstancias: que más de la mitad de las asociaciones no disponen de local, y que un tercio más lo disfruta pero en forma de cesión (lo cual condiciona su uso e independencia); tan sólo nos queda otro tercio del total de las asociaciones en activo que disfruta de manera autónoma de local para su funcionamiento, tanto ordinario como para la organización de actividades extraordinarias o complementarias. Sin seguir ningún tipo de determinismo, sí queremos ver aquí un elemento explicativo tanto de la debilidad y dependencia de las AA. VV. como de sus limitaciones en cuanto a los cauces y formas de sociabilidad que puedan desarrollar. De hecho, una exploración estadística más pormenorizada nos deja clara la vinculación significativa que existe entre la disponibilidad de un local y el desarrollo de actividades de servicio directo al barrio, con lo cual se corrobora claramente la relación entre disponibilidad de locales y el carácter de las actividades que se implementan. Es más, a partir de estos datos generales podemos inferir el escaso patrimonio propio del que las asociaciones disponen, ya que, si no son propietarias 0 arrendatarias de un local, difícilmente podrán disponer, por ejemplo, de patrimonio mueble. 
La vida asociativa ordinaria se rige normalmente por dos tipos de contactos. Uno informal, que se produce en la propia interacción de los vecinos en la sede de la asociación (si es que se dispone de la misma, está abierta y supone realmente un espacio de sociabilidad dentro del barrio), y otro formal, que tiene como protagonistas a los socios de la AA. VV. y que se produce sobre la base del propio funcionamiento reglado de la misma. Con el objetivo de reflexionar sobre la vida asociativa de nuestras AA. VV. nos fijamos en las asambleas de socios y en las reuniones de sus juntas directivas. En cuanto a las primeras, el número medio de asambleas celebradas durante el año 2000 no revela precisamente un universo asociativo dinámico, en el que los contactos reglados y los debates formales sobre problemas colectivos marquen la pauta del funcionamiento asociativo vecinal. Más bien al contrario, el número medio de asambleas realizadas nos viene a indicar el cumplimiento estricto del número de sesiones mínimas recogidas en los correspondientes estatutos, y no tanto la idea de la asamblea extraordinaria como foro de debate vecinal en torno a problemáticas colectivas.

\begin{tabular}{|l|c|}
\hline \multicolumn{2}{|c|}{ Número de asambleas año 2000} \\
\hline Ninguna asamblea & $11 \%$ \\
\hline Una asamblea & $52,9 \%$ \\
\hline Dos asambleas & $20,7 \%$ \\
\hline Entre 3 y 6 asambleas & $13,1 \%$ \\
\hline Más de 6 asambleas & $2,4 \%$ \\
\hline
\end{tabular}

En un ámbito como el vecinal, de contacto supuestamente permanente y fluido, y de asuntos y problemáticas que enlazan directamente con la más estricta cotidianeidad, la coordinación y el funcionamiento de las juntas directivas de estas organizaciones supone la piedra angular de todo el funcionamiento asociativo, de su eficacia y funcionalidad. Por eso una referencia al número de reuniones de la junta directiva se nos antoja aún más relevante que el de asambleas anuales. De todos es bien sabido que la participación en este tipo de asociaciones es escasa, y que en la mayoría de los casos son las juntas directivas o sus presidentes los que mantienen vivas a las AA. VV. Por eso la frecuencia de reuniones directivas - una vez asumida la debilidad a otros niveles más amplios- es un indicativo complementario al anterior, e incluso de un valor más definitorio en cuanto al funcionamiento interno de la asociación. 


\begin{tabular}{|l|c|}
\hline \multicolumn{2}{|c|}{ Reuniones junta directiva año 2000} \\
\hline Ninguna & $8,2 \%$ \\
\hline Entre 1 y 6 & $40,6 \%$ \\
\hline Entre 7 y 12 & $29,7 \%$ \\
\hline Entre 12 y 24 & $11 \%$ \\
\hline Más de 24 & $10,5 \%$ \\
\hline
\end{tabular}

Tenemos así que el $84,6 \%$ de las AA. VV. celebran dos o menos asambleas a lo largo del año, momento exclusivo en el que son convocados formalmente la totalidad de los socios. Por otra parte, nos encontramos con que tan sólo en un 21,5\% de las AA. VV. sus juntas directivas se reúnen más de una vez al mes. Con estos datos resulta evidente que la vida asociativa reglada es en general muy débil. Más allá de estos ámbitos, las asociaciones establecen comunicación tanto con sus socios como con los vecinos en general, utilizando para ello -y en cierto modo de manera paradójica - preferentemente los cauces más formalizados, lo cual, pensamos, agudiza la lejanía y debilidad que encontramos claramente reflejadas en los datos que se desprenden de nuestro trabajo de campo.

\begin{tabular}{|l|c|l|r|}
\hline \multicolumn{1}{|c|}{ Forma comunicación con los socios } & \multicolumn{2}{c|}{ Forma comunicación con los vecinos } \\
\hline Escritos & $81,2 \%$ & Escritos & $82,2 \%$ \\
\hline Cara a cara & $43,1 \%$ & Cara a cara & $32,6 \%$ \\
\hline Red & $3,4 \%$ & Red & $1,8 \%$ \\
\hline Radio/TV & $10,4 \%$ & Radio/TV & $20,9 \%$ \\
\hline
\end{tabular}

El papel de las mujeres en el ámbito de las relaciones vecinales es muy relevante. No obstante, su presencia y participación efectiva en las organizaciones que tienen precisamente al vecindario como base asociativa es un fenómeno mucho más reciente. Incluso en este contexto encontramos una presencia muy importante de las mujeres a nivel informal que, sin embargo, no tiene su correspondiente traducción en los ámbitos formalizados de esas organizaciones. Las mujeres, de manera general, participan en las actividades asociativas, e incluso son las líderes incuestionables de muchas de éstas, pero su presencia en la estructura organizativa, o mejor dicho, directiva, ha quedado tradicionalmente relegada. Los resultados de nuestro estudio arrojan nuevos datos sobre esta circunstancia en los municipios medios andaluces. La media de miembros de las directivas de estas AA. VV. es de 6,04 . De entre ellos una media de 2,89 serían mujeres, lo que nos sitúa ante un $47,8 \%$ de presencia femenina en las directivas, lo cual puede irnos haciendo desterrar esa 
situación tradicional de mujeres que participan en actividades informales pero que están completamente relegadas del espacio público formalizado. Es cierto que en la mayoría de las ocasiones la presidencia está ocupada por un varón, pero también se detecta que, tanto cuantitativa como cualitativamente, la presencia femenina en este tipo de puestos está claramente en alza.

En nuestro trabajo de campo hemos podido contactar con la práctica totalidad de los presidentes de las AA. VV. activas. Ello nos ha permitido establecer un somero perfil de estos cargos que juegan el papel central en estas organizaciones. Aquí queremos resaltar que el $71,6 \%$ de los mismos se encuentran profesionalmente en activo, resultando curioso - pero previsible- que el $24 \%$ sean pensionistas y jubilados, y que en torno al $5 \%$ sean desempleados. La creciente presencia de "no activos" en estos cargos deberá ser motivo de reflexión y estudio específico.

\subsection{Sobre la articulación externa}

El otro eje sobre el que bascula nuestra caracterización general del asociacionismo vecinal es el de su articulación externa. Esta articulación la entendemos a dos niveles: por un lado, la conexión con otras asociaciones, y por otro, la relación con la administración en general, y muy especialmente la administración y el gobierno local como interlocutor principal de la actividad asociativa vecinal.

En principio, podemos fijarnos en los niveles de organización de actividades que las $\mathrm{AA}$. VV. tienen con otras asociaciones 0 entidades. De esta forma indirecta podemos hacernos una idea de la imbricación real de este movimiento asociativo con otros componentes del sistema asociativo en general y del sistema político local.

\begin{tabular}{|l|l|l|l|}
\hline \multicolumn{4}{c}{ Actividades con otras organizaciones } \\
\hline Sindicatos & $0,5 \%$ & Asoc. de mujeres & $4,4 \%$ \\
\hline Orga. políticas & $1,8 \%$ & Asoc. de jubilados & $3,1 \%$ \\
\hline Instit. religiosas & $2,3 \%$ & Instit. educativas & $5 \%$ \\
\hline Hermandades & $6,8 \%$ & Ayuntamiento & $26,9 \%$ \\
\hline ONG & $3,1 \%$ & AA. VV. & $22,2 \%$ \\
\hline Ecologistas & $3,2 \%$ & Asoc. Deportivas & $3,9 \%$ \\
\hline Instit. Recreat./culturales & $5 \%$ & & \\
\hline
\end{tabular}


Como vemos en este amplio catálogo, las AA. VV. no se caracterizan precisamente por organizar actividades con otras organizaciones. Tan sólo destacan la vinculación con otras AA. WV., y, sobre todo, con respecto a los ayuntamientos. Por encima de todo ello parece establecerse una situación clara de aislamiento de las organizaciones vecinales en cuanto al conjunto del movimiento asociativo. No obstante, las dudas sobre la articulación externa de las AA. VV. y la cohesión con otras organizaciones se disipan en gran parte cuando nos hacemos eco de los datos referidos a la propia articulación de las AA. VV. entre sí.

\begin{tabular}{l|l|l|}
\multicolumn{3}{c}{ Pertenencia a federaciones de $\boldsymbol{A A V V}$} \\
\hline No & $58,2 \%$ \\
\hline Sí & & $41,8 \%$ \\
\hline & Local & $76,2 \%$ \\
\hline Comarcal & $14,4 \%$ \\
\hline & Provincial & $22,2 \%$ \\
\hline & Autonómico & $9,9 \%$ \\
\hline
\end{tabular}

A nuestro juicio, dos datos destacan por encima de los demás: menos de la mitad de las AA. VV. están federadas, y sólo están federadas a nivel autonómico el $4 \%$ de las AA. WV. de las ciudades medias de Andalucía. Estas cifras dan una imagen inequívoca de desarticulación interna del movimiento asociativo vecinal. En cierto sentido, nos hablan más bien de una constelación de asociaciones que, en realidad, no se constituyen propiamente en movimiento, habida cuenta de la falta de cohesión que presentan. Por otro lado, es curioso cómo la articulación (la federación), cuando se da, es a nivel local, y, en todo caso, provincial —probablemente como una consecuencia directa del primer tipo de asociación y no tanto como una voluntad específica - quedando muy relegados los niveles comarcal y autonómico. Esta circunstancia viene a reflejar claramente una de las características de la propia articulación sociopolítica del territorio y la sociedad andaluza, en la que el localismo aparece como un fenómeno muy importante, ahora puesto de manifiesto en cuanto a la federación de asociaciones vecinales.

En contraposición a esta debilidad tanto de la articulación externa como de la cohesión del propio asociacionismo vecinal, la conexión de las organizaciones vecinales con la administración (sobre todo con la local) es notable. El $60 \%$ de las asociaciones activas manifiestan unas buenas relaciones y una valoración muy 
positiva hacia los respectivos equipos de gobierno municipales. Pero más allá de los aspectos meramente valorativos, otras circunstancias apuntan de forma más explícita hacia esa conexión privilegiada. Baste citar algunos ejemplos: el $58 \%$ de las asociaciones en activo han recibido subvención pública durante el año 2000 (esta subvención, o bien es mayoritariamente de fondos locales, o ha sido gestionada desde los ayuntamientos); más del $40 \%$ de las asociaciones disfrutan de un local cedido normalmente por los ayuntamientos; y la media de reuniones de las AA. VV. con representantes municipales está en 5,96, lo cual supone prácticamente contacto formal bimensual.

Estos datos habría que ponerlos en conexión con otros anteriormente referidos para establecer una lectura más completa de la situación. Por un lado, hay que tener en cuenta que hemos encontrado vinculaciones estadísticas significativas entre la obtención de subvenciones y las actividades que se desarrollan. Esto es, mientras que las subvenciones en sí mismas no nos indican el uso preferencial de los fondos recibidos, cuando cruzamos los resultados de "tener subvención" con "actividad central" de la asociación, aparece una clara correlación entre recibir subvención y tener como actividad central la celebración de fiestas y la oferta de servicios comunitarios. Esto, por un lado, nos muestra la vinculación positiva del recibir subvenciones, pero, por otro, también nos indica que no existe esa misma vinculación entre subvención y tener como actividad principal las acciones reivindicativas, por ejemplo. Con ello podemos entrever que las subvenciones oficiales están modelando claramente las actividades de las AA. WV., potenciando unas con respecto a otras.

En otro orden de asuntos, debemos tener también presente la relación detectada entre número de reuniones mantenidas con representantes del ayuntamiento y la valoración que se hace de la institución. Los resultados de la comparación estadística establecen con claridad una correlación entre ambos parámetros: a mayor número de reuniones con los representantes municipales se detecta una mejor valoración de la institución.

No es aventurado por tanto establecer un grado de conexión notable entre el movimiento vecinal y las instituciones municipales. Parece evidente la existencia clara de redes verticales entre ayuntamientos y AA. VV., en detrimento de la articulación de redes horizontales entre las propias AA. VV. (sin haber entrado a valorar cuántas de las federaciones de AA. VV. existentes están animadas por los gobiernos locales). 
Por todo ello, tendemos a calificar a gran parte del movimiento vecinal como vinculado asimétricamente con los gobiernos locales, ya que entendemos que estos últimos tienen un papel fundamental en la vida y actividades asociativas a múltiples niveles.

\section{ASOCIACIONISMO VECINAL Y ESPECIFICIDAD SOCIO- CULTURAL}

En un territorio como el andaluz, la ubicación geográfica —con lo que ello conlleva en cuanto a particularidades sociales, demográficas, económicas y culturales - supone un factor que tradicionalmente ha permitido diferenciar al conjunto de localidades a las que nos estamos refiriendo. En este sentido, hemos dividido a los 40 municipios medios andaluces en cuatro grupos de afinidad sociocultural-geográfica. A saber:

\begin{tabular}{l|l|l|l|}
\hline Costa & Interior & Campiña & Areas metropolitanas \\
\hline Adra & Alcalá la Real & Antequera & Camas \\
\hline Almuñécar & Andújar & Arcos & Coria \\
\hline Benalmádena & Baza & Baena & La Rinconada \\
\hline Barbate & Cabra & Carmona & Mairena del Aljarafe \\
\hline Estepona & Guadix & Écija & Puerto Real \\
\hline Fuengirola & Loja & Lebrija & San Juan de Aznalfarache \\
\hline Mijas & Martos & Los Palacios & San Roque \\
\hline Rincón de la Victoria & Priego de Córdoba & Lucena & \\
\hline Roquetas de Mar & Puente Genil & Montilla & \\
\hline Rota & Ronda & Morón & \\
\hline Torremolinos & Úbeda & Utrera & \\
\hline
\end{tabular}

Aunque nos hemos basado en datos cuantitativos y de ubicación geográfica para establecer esta agrupación, quizá el principal argumento que la justifica es de carácter cualitativo: resulta evidente que las localidades agrupadas de esta forma comparten afinidades culturales, sociales, económicas, geográficas e históricas. Lo que pretendemos, lejos de proponer una clasificación de municipios con intenciones generalizadoras, es explorar si estas afinidades se traducen o no en vinculaciones caracteriológicas entre las AA. VV. Para ello realizamos una comparación entre los rasgos asociativos vecinales de los municipios así agrupados con los rasgos generales analizados anteriormente. De esta forma, atendemos a las desviaciones 
caracteriológicas que se detectan en los grupos costa, interior, campiña y áreas metropolitanas con respecto a las asociaciones en su conjunto. Pero antes de emprender esta reflexión, quizá sea oportuno considerar una serie de parámetros generales de esta tipologización de municipios que la reforzaría como referente comparativo.

\begin{tabular}{|l|c|c|c|c|c|}
\hline Zona & Población & Municipios & $\boldsymbol{N}^{\boldsymbol{o}} \boldsymbol{A} \boldsymbol{A} . \boldsymbol{V} \boldsymbol{V}$ & \% A A. $\boldsymbol{V} \boldsymbol{V}$ & Hab./AA. $\boldsymbol{V V}$. \\
\hline Costa & 341.150 & 11 & 95 & $24,8 \%$ & 3.591 \\
\hline Interior & 279.979 & 11 & 65 & $17 \%$ & 4.307 \\
\hline Metropolitana & 184.051 & 7 & 65 & $17 \%$ & 2.831 \\
\hline Campiña & 339.205 & 11 & 158 & $41,2 \%$ & 2.146 \\
\hline Total & 1.144 .385 & 40 & 383 & $100 \%$ & 2.988 \\
\hline
\end{tabular}

Analizaremos ahora cada una de las zonas propuestas resaltando exclusivamente aquellos factores que las particularizan con respecto al conjunto de ciudades medias andaluzas.

\subsection{Costa}

El primer aspecto que destaca dentro de esta zona es que las AA. VV. de la costa se ubican con mayor frecuencia fuera del núcleo principal de población de sus municipios. Esta zona es un área de fuerte crecimiento demográfico y expansión urbanística, lo que explica que más del $60 \%$ de sus AA. VV. estén ubicadas en entidades locales menores, barriadas rurales y urbanizaciones. Este factor de "lejanía" con respecto a la cabecera municipal y las instituciones allí ubicadas no es sólo espacial. Así, resulta curioso que en esta área se detecte el menor índice de presencia de las AA. VV. en los registros locales $(74,7 \%)$, que antes hemos calificado como los más fiables de entre los manejados en nuestro trabajo. Pero además, las asociaciones de la costa son las que disfrutan de menos subvenciones (tan sólo el 20\% de las mismas tienen alguna ayuda económica, frente al $42 \%$ de la muestra general). Así mismo, es menos frecuente que las AA. VV. costeras organicen actividades con los ayuntamientos (15,8\%, frente al $26,9 \%$ general). También la valoración que hacen de las relaciones con los ayuntamientos es ligeramente inferior que las de las demás organizaciones vecinales. Con todo ello, es lógico concluir que la principal característica del asociacionismo vecinal costero es precisamente su distanciamiento de los gobiernos locales en diferentes aspectos, como acabamos de comprobar. Para 
afianzar aún más esta afirmación, habría que destacar que sus actividades centrales tienen menos que ver con la organización de fiestas ( $43,2 \%$, frente al $59 \%$ general), y mucho más con actividades reivindicativas ( $55,8 \%$ frente al 36,8 general). No es difícil imaginar que la mayoría de estas actividades de reivindicación se orienten hacia los gobiernos locales.

Por lo demás, debemos tener en cuenta que se agudizan la desarticulación y el aislamiento de las AA. VV. en tanto en cuanto se reducen mucho las ya de por sí débiles conexiones de las $A A$. VV. entre sí: la organización de actividades de unas AA. VV. con otras cae hasta el $12,6 \%$, cuando la tendencia general engloba al $22,2 \%$ de las organizaciones. Del mismo modo, la participación de las AA. VV. costeras en federaciones de AA. VV. es, con mucho, la más baja de los cuatro grupos sociogeográficos que hemos considerado (tan sólo el 17,5\% de estas asociaciones están federadas, cuando la media global se sitúa en torno al $42 \%)$. Curiosamente, e invirtiendo una tendencia general, las AA. VV. costeras que sí están federadas lo hacen en mayor proporción en federaciones de carácter provincial (58\%) que en federaciones locales $(35,3 \%)$, acentuando aún más esa desconexión con el ámbito local al que antes nos referíamos.

\subsection{Interior}

En esta zona encontramos una serie de desviaciones respecto a la caracterización general que nos presentan un movimiento vecinal de características muy particulares e integradas. En la zona interior de Andalucía encontramos el mayor número proporcional de AA. WV. que disfrutan de subvención oficial $(52,3 \%)$, las que en mayor proporción se dedican a la organización de fiestas y celebraciones (83\%), las que menor número de asambleas celebran anualmente (el 78,5\% sólo celebraron una o ninguna durante el año 2000), las que en mayor proporción organizan actividades conjuntamente con hermandades $(13,8 \%)$, y las que tienen menor proporción de mujeres en sus juntas directivas (22,5\%, frente al $47,8 \%$ general). Junto con a todo ello, las AA. VV. del interior son las que menor número de reuniones mantienen con representantes municipales (una media de 3,91 por asociación, mientras que la muestra general presenta una media de 5,96$)$.

Como vemos, este conjunto de parámetros nos habla de un asociacionismo muy subvencionado y centrado en las actividades lúdicas (que explica incluso la mayor 
vinculación a hermandades), con escasa vida asociativa y una anacrónicamente baja presencia femenina en sus órganos de gestión.

\subsection{Areas metropolitanas}

Por su parte, las asociaciones vecinales ubicadas en las zonas metropolitanas andaluzas ofrecen un perfil hasta cierto punto antitético con respecto al de las del interior. En principio, presentan una menor presencia en los registros autonómicos $(72,3 \%)$ que las de otras zonas, mientras que, por el contrario, su reflejo en los registros locales es mayor que la media $(93,8 \%)$. En las zonas metropolitanas encontramos las AA. VV. más antiguas del conjunto andaluz analizado: el $54,2 \%$ de las asociaciones nacieron con anterioridad a 1991, mientras que este grupo en la muestra general se reduce al $41,5 \%$.

En cuanto a los motivos de su aparición, es destacable que la organización de fiestas y celebraciones sea sensiblemente menor a la media $(4,6 \%$, frente al $8,3 \%$ general), mientras que las razones en torno a problemas urbanísticos y de vivienda es mayor (49,2\%, frente al $40,1 \%$ general). En las áreas metropolitanas se detecta la mayor concentración proporcional de asociaciones que disponen de local ( $70 \%$, frente al $48,6 \%$ general), así como de locales en propiedad $(27,3 \%$, frente al $13 \%)$. Las actividades centrales en la actualidad presentan igualmente una primacía de las fiestas y celebraciones, aunque ligeramente matizadas a la baja respecto a los resultados medios, mientras que sí resulta llamativo el número de AA. WV. que se dedican fundamentalmente a los servicios comunitarios (53,8\%, frente al $41,8 \%$ general).

Otro apartado en que estas AA. VV. de las áreas metropolitanas marcan una diferencia con el conjunto de asociaciones es el referido a su funcionamiento interno. El número de reuniones de las juntas directivas: (el $73,1 \%$ de las AA. WV. celebran más de 6 reuniones anuales -en la muestra total sólo el 51\%-), y que el porcentaje de mujeres en las juntas directivas llegue hasta el 60,6\% (en el general es del 47,8\%) nos habla de un sistema asociativo más activo y dinámico que el del conjunto de la muestra analizada. 
Pero además de todo ello, habría que apuntar una mayor imbricación de estas asociaciones tanto con otras entidades, y en este sentido es significativo su mayor relación con las ONG (12,3\%, frente al escaso 3,1\% general), como con los propios ayuntamientos (habida cuenta de la media de reuniones con sus representantes: 12,3 reuniones de media, frente a las 5,96 de la muestra general). Así mismo, las AA. VV. de las áreas metropolitanas presentan el mayor índice de pertenencia a federaciones de AA. VV. (61,5\%, cuando la media se sitúa en torno al $42 \%)$, y en su mayoría las federaciones de referencia son de índole local $(85,7 \%)$.

Con todos estos datos no es arriesgado afirmar que en los municipios medios de las áreas metropolitanas andaluzas encontramos el mayor desarrollo y consolidación de las AA. VV. y del movimiento vecinal, sobre todo si tenemos en cuenta la debilidad general de la que partimos.

\subsection{Campiña}

El área que aquí denominamos Campiña es en cierto modo la que marca la pauta estadística para el conjunto, ya que concentra el mayor número de AA. VV. Por eso es la que menores desviaciones presenta respecto a la caracterización general. No obstante, sí resulta destacable la baja proporción de habitantes por asociación -2.146 , frente a la media de 2.988 -, lo que nos habla de un movimiento vecinal realmente importante, al menos desde el punto de vista cuantitativo.

El asociacionismo vecinal en la campiña es el que mayor concentración presenta en los núcleos principales (76\%, frente al $66 \%$ general). También resulta ser el más joven, ya que el $64 \%$ de las AA. VV. nacieron en la década de los noventa (el $57,8 \%$ del general). Igualmente, las AA. VV. de la campiña constituyen un conjunto de organizaciones notablemente subvencionado por instituciones oficiales (el 50\% disfruta de subvención, frente al $42 \%$ de la muestra general), por lo cual no es de extrañar que sea en esta zona donde mejor se valoran las relaciones con los ayuntamientos. Por último, resulta llamativa la altísima presencia femenina en los órganos de gestión de las AA. W.: el 61,3\% de los miembros de las juntas directivas son mujeres (frente al $47,8 \%$ en el general). 


\section{CARACTERIZACIÓN EN VIRTUD DE LAS ESPECIFICIDADES POLIITICAS LOCALES}

Creíamos que sería relevante atender a la configuración de los gobiernos locales como variable ilustrativa de las características de las AA. VV. Ya expusimos en la presentación nuestros presupuestos de partida. Uno de ellos era la vinculación entre el movimiento vecinal, la política local en general, y más concretamente la conformación y el funcionamiento del gobierno local. Estas vinculaciones pueden ser de muy diferente signo: contestación, resistencia, dependencia... Por eso pretendíamos comprobar si las características de las AA. VV. variarían en virtud del carácter del gobierno local en cada caso. Obviamente, la casuística y evoluciones políticas de los cuarenta municipios que estamos analizando son muy dispares, y casi podemos decir que cada uno de ellos supone un universo político en sí mismo, lo que ha quedado patente en las etnografías que hemos realizado. No obstante, hemos creído oportuno considerar ciertas homogeneidades, estableciendo tres grupos de municipios a tenor de los resultados electorales locales desde 1979. De nuevo en esta tipologización de municipios se ha mezclado un criterio meramente cuantitativo -en cuanto a número de concejales y constitución de gobierno-, con otro cualitativo en torno al concepto de hegemonía política. Concretamente, hemos determinado que en una localidad existe hegemonía política cuando en las tres últimas legislaturas se ha dado una mayoría absoluta a favor de un mismo partido político, o al menos dos mayorías absolutas y una relativa. Ha surgido así un grupo conformado por localidades donde existe una hegemonía, del PSOE, otro para localidades con hegemonía de otras formaciones políticas (PP, PA o IU-CA), y un tercero para localidades donde no existe hegemonía sino más bien un sistema pluralista del gobierno local sostenido en el tiempo. 


\begin{tabular}{|l|l|l|l|}
\hline Grupo A (Hegemonía PSOE) & Grupo B (pluralidad) & Grupo C (Hegemonía de otros partidos) \\
\hline Alcalá la Real & Adra & Almuñécar \\
\hline Andújar & Arcos & Cabra \\
\hline Antequera & Barbate & Fuengirola \\
\hline Baena & Benalmádena & Puerto Real \\
\hline Baza & Carmona & Torremolinos \\
\hline Camas & Coria & \\
\hline Guadix & Écija & \\
\hline La Rinconada & Estepona & \\
\hline Lebrija & Loja & \\
\hline Los Palacios & Lucena & \\
\hline Mairena del Aljarafe & Montilla & \\
\hline Martos & Morón & \\
\hline Mijas & Puente Genil & \\
\hline Priego de Córdoba & Rincón de la Victoria \\
\hline San Juan de Aznalfarache & Ronda & \\
\hline San Roque & Roquetas de Mar \\
\hline Utrera & Rota & \\
\hline & Úbeda & \\
\hline
\end{tabular}

Nuestra pretensión era comprobar si las diferentes circunstancias políticas municipales constituyen un factor que propicia la caracterización diferencial del asociacionismo vecinal. Esto significa presuponer a un nivel más general que (1) los distintos partidos tienen y desarrollan políticas sobre asociacionismo diversas; y (2) que la forma de ejercer el gobierno municipal —hegemonía o pluralidad-es un factor clave en las relaciones con la ciudadanía. Tal como hicimos en el epígrafe anterior, comparamos los resultados de los diferentes items generales con los resultados particularizados. Fijándonos en las desviaciones, significativas podremos argumentar en torno a una hipotética relación de las características de las AA. VV. con la forma en que se gobierna la localidad.

Pero antes de entrar en el análisis, quizá sea oportuno pararnos un instante a comprobar las magnitudes y dimensiones de los grupos de municipios que hemos creado en virtud de su funcionamiento político local. 


\begin{tabular}{|l|c|c|c|c|c|}
\hline Tipos & Población & Municipios & $\boldsymbol{N}^{\boldsymbol{o}} \boldsymbol{A} \boldsymbol{A} . \boldsymbol{V} \boldsymbol{V}$ & $\boldsymbol{\%} \boldsymbol{A A .} \boldsymbol{V} \boldsymbol{V}$ & $\boldsymbol{h a b . / A V V}$. \\
\hline Grupo B & 515.336 & 18 & 184 & $48 \%$ & 2.800 \\
\hline Grupo A & 471.659 & 17 & 157 & $40,9 \%$ & 3.004 \\
\hline Grupo C & 157.390 & 5 & 42 & $10,9 \%$ & 3.747 \\
\hline Total & 1.144 .385 & 40 & 383 & $100 \%$ & 2.988 \\
\hline
\end{tabular}

Los resultados de la exploración estadística siguiendo la hipótesis que planteamos han sido ciertamente difusos. Las desviaciones que presentan los tres grupos de localidades en virtud de su configuración política no son demasiado relevantes. En concreto, las AA. VV. que se ubican en los grupos A y $B$ son, en líneas generales, muy similares en su perfil y -obviamente- las diferencias con los resultados de la muestra general son muy escasas. Por eso vamos a desistir aquí de hacer una caracterización específica de cada grupo de municipios/asociaciones. Sin embargo, aparecen algunos rasgos que sí merecen ser destacados.

Las fechas de aparición de las AA. VV. en los grupos A y B se corresponden básicamente entre sí y con respecto a los resultados generales. No obstante, el perfil de este item varía sustancialmente para las asociaciones que se ubican en municipios con hegemonía de otros grupos políticos distintos del socialista. En estos casos las asociaciones son sensiblemente más antiguas. Para municipios de los grupos A y $\mathrm{B}$, las AA. W. han aparecido en un $60 \%$ después de 1990. En los municipios gobernados hegemónicamente por otros partidos, la tendencia se invierte, las asociaciones de la última década constituyen un $39 \%$, mientras que el $61 \%$ restante son $\mathrm{AA}$. WV. que surgieron a lo largo de los ochenta e incluso con anterioridad.

Con respecto al motivo de aparición, habría que destacar el bajo porcentaje de AA. VV. que surgen por razones de urbanismo y vivienda en los municipios con hegemonía socialista, y así mismo el escaso número de asociaciones que aparecen con el objetivo de fomentar manifestaciones festivas en los municipios del grupo $B$ $(3,9 \%)$. Esta casuística se manifiesta de manera similar cuando nos ocupamos de las actividades centrales de las AA. VV. en la actualidad. Así, las AA. VV. de municipios gobernados hegemónicamente por el PSOE son las que en mayor proporción se dedican a organizar fiestas y celebraciones (un $70 \%$ ), mientras que las AA. VV. de municipios del grupo $B$ —de gobierno local más pluralizado— serán las que en mayor proporción centren sus esfuerzos en actividades reivindicativas (39,1\%), aunque no con una desviación notablemente significativa. 
La tenencia de local y el disfrute de subvenciones sigue una tendencia parecida a la vista hasta ahora, en la que los municipios de los grupos A y B se asemejan bastante entre sí, mientras que la hegemonía de otros partidos suponen una desviación de rasgos hasta cierto punto significativa. En este sentido, es notable el número de AA. VV. que disponen de local $(57,1 \%$, frente al 48,6 de la muestra general) y la proporción que lo disfrutan en propiedad (45,8\%, frente al $13 \%$ del conjunto) en los municipios gobernados hegemónicamente por otros partidos distintos del socialista. Lo mismo ocurre con las subvenciones, ya que en estas situaciones de hegemonía no socialista encontramos la mayores proporciones de asociaciones subvencionadas $(57,1 \%$, frente al $42 \%$ de la muestra general).

En cuanto a la participación de las AA. VV. en federaciones, encontramos que en las ubicadas en municipios del grupo $B$ los índices arrojan unos resultados prácticamente análogos a los del conjunto de las AA. VV. en Andalucía $(41,7 \%$ sí pertenecen a federaciones y el $58,3 \%$ no). Las matizaciones vienen ilustradas en los municipios con hegemonía socialista, en los que el nivel de federación es sensiblemente menor $(35,6 \%)$. Pero sobre todo los datos más relevantes nos los proporcionan las AA. VV. de los municipios con hegemonía política de partidos distintos al PSOE (grupo C), donde encontramos un notablemente mayor índice de pertenencia a federaciones de AA. WV., concretamente el $62 \%$ (recordemos que la media se sitúa en torno al $42 \%$ ).

Los últimos elementos destacables son la proporción de mujeres en las juntas directivas y el número de reuniones con responsables municipales. En ambos se revela de nuevo una desviación de la media para el grupo de hegemonía no socialista, mientras que los grupos A y B no presentan variabilidad ni entre sí, ni con respecto a la muestra general. Las AA. VV. de estos cinco municipios que integran el grupo $\mathrm{C}$, "hegemonía de otros partidos", tienen el mayor porcentaje de mujeres en las juntas directivas (58,1\%, frente al $47,8 \%$ de la muestra general), y mantienen una media de 12,57 reuniones anuales con responsables municipales, cuando la media del conjunto es de seis.

Con estas ligeras variaciones podríamos concluir en una diferenciación aunque somera - del asociacionismo en municipios gobernados hegemónicamente por partidos distintos del socialista que apunta hacía una política más activa de fomento y sustento de un asociacionismo vecinal, que además tienen mayor 
tradición y antigüedad. Por otra parte, resulta muy relevante la escasa diferenciación en la caracterización de las AAVV que se ubican en municipios gobernados hegemónicamente por el PSOE o bien con una política local de carácter más pluralista: no parece que el gobierno consistente y mantenido a lo largo del tiempo propicie, para el caso del PSOE, un modelo de asociacionismo vecinal particular. En general, parece que ni el signo, ni el tono del gobierno municipal se revelan como factores explicativos centrales de la caracterización del asociacionismo vecinal en los municipios estudiados.

\section{CONCLUSIONES}

El asociacionismo vecinal en las ciudades medias andaluzas está sujeto a un notable sobredimensionamiento por parte de las administraciones. Esta circunstancia es muy problemática si tenemos en cuenta que un $44 \%$ de las asociaciones vecinales incluidas en los distintos registros administrativos no existen realmente.

Más de la mitad de las asociaciones de vecinos tiene un origen muy reciente - en la década de los noventa-, por tanto prácticamente desvinculado del asociacionismo que protagonizó la transición democrática. El movimiento vecinal se ocupa principalmente de la organización de celebraciones y fiestas, y menos de la mitad de las AA. VV. tienen como objeto principal la reivindicación. El funcionamiento interno de las asociaciones es francamente débil, como se desprende de la escasa sociabilidad formal e informal que patrocinan: muy bajo número de asambleas anuales, escasa frecuencia de reuniones de las directivas y cauces muy indirectos de conexión con los vecinos. A todo ello se le suma como agravante el que menos de la mitad de las asociaciones dispongan de local. No obstante, habría que destacar que casi el $50 \%$ de los cargos directivos están ocupados por mujeres, lo cual confirma nuevas tendencias en la participación asociativa.

Las AA. W. están claramente desvinculadas del conjunto del sistema asociativo del que forman parte en cada localidad. Así mismo se detecta una escasa cohesión como movimiento, evidenciada, entre otros factores, en el bajo índice de federación interna. Estas dos circunstancias restan significación y potencialidad sociopolítica a las asociaciones de vecinos de los municipios medios andaluces. Al mismo tiempo se evidencia una fuerte dependencia del asociacionismo vecinal con respecto a 
los gobiernos locales, corroborada en distintos aspectos (locales, subvenciones, valoraciones, conexiones...). Todo ello nos presenta el retrato de un tejido asociativo pobre que, por otra parte, y paradójicamente, goza de un notable reconocimiento formal e institucional como cauce de participación social y de articulación de la sociedad civil con las distintas instancias del Estado.

Nuestra búsqueda de significaciones con respecto a los factores socioeconómico-culturales y político-locales presenta conclusiones bien distintas. Mientras que el tono y signo de los gobiernos locales se ha mostrado muy débilmente significativo, los criterios socio-culturales-geográficos manifiestan una notable significación, llegándose a establecer claras tipologías asociativas en virtud del contexto geográfico y socioeconómico en el que se ubican las AA. VV. Para nosotros estas circunstancias vienen a evidenciar el carácter marcadamente local de las características del asociacionismo vecinal. Esto es, que las características de las asociaciones vecinales en las ciudades medias de Andalucía están más condicionadas por la propia base sociocultural en la que se insertan que por tendencias y estrategias vinculadas al signo político o al carácter de los gobiernos locales. De este modo, resulta muy elocuente que las asociaciones sean más parecidas/diferentes entre sí en relación con los contextos socioculturales en los que operan que en relación con el signo del gobierno y el ambiente político local de los que forman parte. Esta conclusión nos obligará a reflexionar sobre las preconcepciones que vinculan prioritariamente las características del asociacionismo ciudadano con el contexto político en el que se inserta.

\section{BIBLIOGRAFÍA}

CANO GARCÍA, G. y GARCÍA GÓMEZ, A. (2000) "Sistema de ciudades andaluzas". En Cano, G. (Dir.) Conocer Andalucía. Gran Enciclopedia Andaluza del Siglo XXI. Vol. 5: Infraestructuras y Ordenación del Territorio. Sevilla, Ediciones Tartessos, pp. 11-71.

CARMONA GALLEGO, J. y COLLADO BRONCANO, M. (1994) "¿Por dónde anda hoy el asociacionismo en Andalucía?". En Documentación Social, n 94, monográfico dedicado al Mundo Asociativo. 
DEL RÍO SÁNCHEZ, A. y COCA PÉREZ, A. (2003) "Poder y Movimiento Ciudadano en Andalucía. Las asociaciones de vecinos en ciudades del medio rural y del litoral andaluz", en Actas del IX Congreso de Antropología de la Federación de Asociaciones de Antropología del Estado Español, Barcelona: Institut Català d'Antropologia.

ESCALERA REYES, J. (1988) "El tópico de la debilidad asociativa andaluza desde la Antropología Social". Revista de Estudios Andaluces, n 11, pp. 87-108.

- (2001a) "Formas de sociabilidad. Fiestas y Religiosidad". En Cano, G. y otros, La Identidad del Pueblo Andaluz. Sevilla, Defensor del Pueblo Andaluz, pp. 133-143.

_-(2001b) "Sociabilidad, relaciones de poder y cultura política". En G. Cano (dir.) Conocer Andalucía. Gran Enciclopedia Andaluza del Siglo XXI. Tomo 7, Sevilla, Ediciones Tartessos, pp. 124-163.

—(2002) "Asociacionismo civil". En S. Rodríguez (coord.) Proyecto Andalucía. Vol. 5: Sociedad, Sevilla Publicaciones Comunitarias, S.A. Grupo-Hércules, pp. 310-338.

ESCALERA REYES, J. Y MARTíN DÍAZ, E. (1990) "Antropología y ciudades: aportaciones al análisis de la especificidad urbana andaluza". En Jornadas sobre Ciudades Medias. Sevilla, Consejería de Obras Públicas y Transportes, pp. 95-102.

FERIA TORIBIO, J. M. (1990) "Las ciudades medias: perspectivas y retos". En Jornadas sobre Ciudades Medias. Sevilla, Consejería de Obras Públicas y Transportes, pp. 31-36.

- (1992) El sistema urbano andaluz: aglomeraciones urbanas, áreas de centralidad y ámbitos desarticulados. Sevilla, Instituto de Desarrollo Regional-Consejería de Obras Públicas y Transportes.

LÓPEZ CASERO, F. (comp.) (1989) La agrociudad mediterránea: estructuras sociales y procesos de desarrollo. Madrid, Ministerio de Agricultura, Pesca y Alimentación.

LÓPEZ CHACÓN, R. (1990) "Las ciudades medias andaluzas". En Jornadas sobre Ciudades Medias. Sevilla, Consejería de Obras Públicas y Transportes, pp. 69-74.

NAVARRO YÁÑEZ, C. (1999) El sesgo participativo. Innovación democrática en municipios del sur de Europa (1960-1995). Córdoba, CSIC. 
RUIZ BALLESTEROS, E. (2000) Construcción simbólica de la ciudad. Política local y localismo. Madrid-Buenos Aires, Miño y Dávila Editores.

RUIZ BALLESTEROS, E. y PÁEZ SOTO, C.(1997) "Aproximación al movimiento vecinal y la cultura política en Jerez". Demófilo. Revista de Cultura Tradicional de Andalucía, $n^{0} 34$, pp. 113-137.

W. AA. (1994) Documentación Social, nº 94, monográfico dedicado al Mundo Asociativo.

ZOIDO NARANJO, F. (1990) "Las ciudades medias en Andalucía". En Jornadas sobre Ciudades Medias. Sevilla, Consejería de Obras Públicas y Transportes, pp. 13-28. 